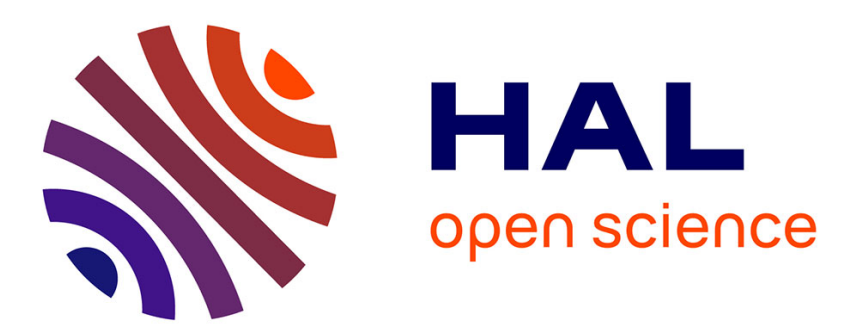

\title{
Efficient hybrid method for the modal analysis of optical microcavities and nanoresonators
}

Philippe Lalanne, T. Wu, D. Arrivault, M. Duruflé, A. Gras, F. Binkowski, S. Burger, W. Yan

\section{- To cite this version:}

Philippe Lalanne, T. Wu, D. Arrivault, M. Duruflé, A. Gras, et al.. Efficient hybrid method for the modal analysis of optical microcavities and nanoresonators. Journal of the Optical Society of America. A Optics, Image Science, and Vision, 2021, 38 (8), pp.1224. 10.1364/JOSAA.428224 hal-03358012

\section{HAL Id: hal-03358012 \\ https://hal.science/hal-03358012}

Submitted on 29 Sep 2021

HAL is a multi-disciplinary open access archive for the deposit and dissemination of scientific research documents, whether they are published or not. The documents may come from teaching and research institutions in France or abroad, or from public or private research centers.
L'archive ouverte pluridisciplinaire HAL, est destinée au dépôt et à la diffusion de documents scientifiques de niveau recherche, publiés ou non, émanant des établissements d'enseignement et de recherche français ou étrangers, des laboratoires publics ou privés. 


\title{
Efficient hybrid method for the modal analysis of optical microcavities and nanoresonators
}

\author{
T. Wu, ${ }^{1}$ D. Arrivault, ${ }^{1}$ (i) M. Duruflé, ${ }^{2}$ A. Gras,,${ }^{1,2}$ F. Binkowski, ${ }^{3}$ (i) S. Burger, ${ }^{3,4}$ (i) \\ W. YAN, ${ }^{5,6}$ AND P. LALANNE ${ }^{1, *}$ (i) \\ 'LP2N, Institut d'Optique Graduate School, CNRS, Univ. Bordeaux, 33400 Talence, France \\ ${ }^{2}$ INRIA Bordeaux Sud-Ouest, Talence 33405, France \\ ${ }^{3}$ Zuse Institute Berlin, 14195 Berlin, Germany \\ ${ }^{4} \mathrm{JCM}$ wave $\mathrm{GmbH}, 14050$ Berlin, Germany \\ ${ }^{5}$ Key Laboratory of 3D Micro/Nano Fabrication and Characterization of Zhejiang Province, School of Engineering, Westlake University, \\ 18 Shilongshan Road, Hangzhou 310024, Zhejiang Province, China \\ ${ }^{6}$ Institute of Advanced Technology, Westlake Institute for Advanced Study, 18 Shilongshan Road, Hangzhou 310024, Zhejiang Province, China \\ *Corresponding author: philippe.lalanne@institutoptique.fr
}

Received 19 April 2021; revised 29 June 2021; accepted 30 June 2021; posted 1 July 2021 (Doc. ID 428224); published 26 July 2021

\begin{abstract}
We propose a novel hybrid method for accurately and efficiently analyzing microcavities and nanoresonators. The method combines the marked spirit of quasinormal mode expansion approaches, e.g., analyticity and physical insight, with the renowned strengths of real-frequency simulations, e.g., accuracy and flexibility. Real- and complex-frequency simulations offer a complementarity between accuracy and computation speed, opening new perspectives for challenging inverse design of nanoresonators. (-2021 Optical Society of America
\end{abstract}

https://doi.org/10.1364/JOSAA.428224

\section{INTRODUCTION}

Plasmonic and Mie resonators that confine light in tiny volumes play an essential role in nanophotonics [1]. With the recent deployment of inverse design and artificial intelligence algorithms, there are new opportunities for their design and optimization in enlarged parameter spaces without a priori information on materials and geometries [2-4]. However, the design is computationally expensive and timeconsuming because nanoresonator modeling requires solving Maxwell's equations. In typical settings, for example, using the adjoint-based optimization method, hundreds to thousands of electromagnetic simulations are needed before convergence is achieved [2]. Deep-learning algorithms allow navigating toward a nearly optimum design almost instantaneously by querying the generalization capabilities of neural networks [5-7]. However, to train the network, a huge data set of instances of optical responses for a myriad of structures needs to be generated [3]. More importantly, inverse design methods and deep-learning algorithms often operate as "black boxes" and do not provide physical insight into the designed structure. Complementary approaches, with a better balance between physics and numeric, are desirable.

Here lies the worth of the present hybrid method that combines real- and complex-frequency simulations to enable an accurate and effective analysis of microcavities and nanoresonators. The complex-frequency simulations provide analyticity and physical insight by intuitively considering the dominant natural resonances, e.g., localized surface plasmon modes for metallic nanoantennas, Mie leaky modes for dielectric resonators, or whispering gallery modes for microdisks. These resonances are referred to as quasinormal modes (QNM) in the following and are computed by solving the source-free Maxwell's equations at complex frequencies [1].

The QNM machinery does not have only advantages. For nanoresonators on metal substrates or gratings, high accuracy may be achieved only by considering a large set of modes [8-11]. The latter include QNMs with frequencies far away from the spectral window of interest, numerical modes that originate from the discretization and the truncation of the perfectly matched layer (PML) $[8,9]$, static modes at null frequency $[9,12,13]$, or accumulation points encoding material resonances $[9,14]$. All these modes are quite unimportant for some applications, e.g., nonlinear harmonic conversion $[15,16]$ or structural color generation $[17,18]$. However, for other studies, e.g., predicting the quantum yields of emitters coupled to nanoantennas or the nonlinear dynamics behavior of optomechanical systems $[19,20]$, a quantitative knowledge of the coupling to numerical modes and QNMs outside the spectral range is essential. In the hybrid approach, we get around the necessity to consider many modes by complementing the QNM analysis with a few additional computational results performed at real frequency. The principle is that, in typical cases, the impact of the contribution of numerical modes and QNMs laying outside the spectral window of interest can be accurately interpolated in the spectral domain from a few interpolation points. 
The idea is certainly not new. For instance, it is deeply rooted in the Riesz projection method [21,22], which exploits Cauchy's residue theorem to show that all the salient features of a spectral response can be captured with a restricted set of dominant QNMs and the nonresonant contribution accessed by contour integrals in the complex frequency plane. Hereafter, we rather propose to compute the nonresonant contribution directly at few real frequencies and to perform an interpolation. This offers a balance between analytical description and direct numerical computation, which not only preserves insight into the modal expansion method but also guarantees high accuracy of the computed results.

Our paper is organized as follows: Section 2 offers a pedagogical step-by-step presentation. In Section 3, we give details on the implementation of the hybrid scheme and describe the toolbox that we have incorporated into the freeware MAN [23]. We also test the convergence performance for several interpolation schemes. Section 4 is devoted to a real-size test that is often encountered in nanophotonics. From the test, we quantify the computational speed and discuss advantages. Section 5 concludes the work.

\section{STEP-BY-STEP PRESENTATION OF THE METHOD}

To simply present the method, we consider a hybrid dielectricmetal resonator composed of a silicon cylinder $2 \mathrm{~nm}$ above a $5 \mathrm{~nm}$ thick silver film. The system is excited by a $z$-polarized dipole emitter placed at an off-axis coordinate, shown with the red arrow in Fig. 1(a). The structure supports a wealth of hybrid plasmonic modes offering ultratight field confinement in the gap.

We choose this particular structure not only owing to its potential for studying novel light-matter interaction phenomena [26] but also owing to its relevancy to test the efficiency of the present method. First, the reconstruction accuracy is particularly impacted by QNMs that lie outside the visible frequency range of interest. This property is not encountered with classical metallic-nanoparticle-on-metal geometries; it results from the significant increase of the refractive index of $\mathrm{Si}$ in the blue and ultraviolet, which enables higher-order FabryPerot resonances at high frequencies with Si-insulator-Ag gap plasmons. Second, owing to the presence of the silver film, the Green's tensor has branch cuts in the complex frequency plane and many numerical modes in addition to the QNMs to be considered [1].

\section{A. QNM Reconstruction at Complex Frequencies}

To reconstruct the optical response of the system, we first compute the QNMs. We use the QNMEig solver [9] of the freely available package modal analysis of nanoresonators (MAN) [23], which relies on the finite element method implemented in COMSOL Multiphysics. The solver normalizes the QNMs with the PML-normalization method [24]. COMSOL Multiphysics uses the ARPACK package [27] for computing eigenvectors of large sparse or structured matrices. The package is developed based on the implicitly restarted Arnoldi method, (a)

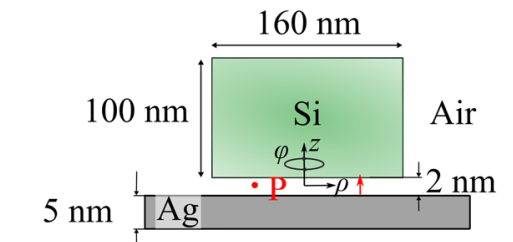

(b)
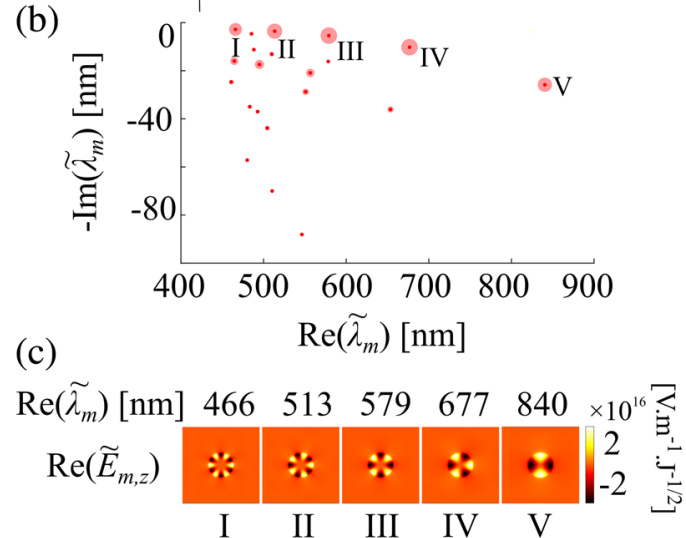

Fig. 1. Set of QNMs excited by a dipole emitter for a hybrid dielectric-metal resonator. (a) Sketch of nanocavity geometry chosen to test the capability of the approach. It is formed by a silicon nanocylinder (diameter $160 \mathrm{~nm}$, height $100 \mathrm{~nm}$ ) on a $5 \mathrm{~nm}$ thick silver film with a gap $g=2 \mathrm{~nm}$. The $z$-oriented dipole emitter (the red arrow) is located in the middle of the gap and at $\rho=53 \mathrm{~nm}$ from the axisymmetry axis. $P$ denotes the symmetric point. (b) Eigenfrequencies in the complex plane computed with the QNMEig solver of MAN. Size of the markers visualizes the spectrally integrated excitation coefficient, $\bar{A}_{m}=\int_{\lambda_{s}}^{\lambda_{e}}\left|\alpha_{m}\left(\frac{2 \pi c}{\lambda}\right)\right|^{2} \mathrm{~d} \lambda$ from $\lambda_{s}=450 \mathrm{~nm}$ to $\lambda_{e}=850 \mathrm{~nm}$. The five dominant (physical) QNMs offer the largest $\bar{A}_{m}$ and are unambiguously identified by changing the PML parameters [9]. They are labelled with Roman numbers, I, II ... (c) Electric field $\operatorname{Re}\left(\tilde{E}_{m, z}\right)$ in the center of the gap $(z=0)$ of the five dominant QNMs with the largest contributions for the reconstruction. QNM near-fields are normalized with the PML method [24]. The permittivity of $\mathrm{Si}$ is given by a multipole Lorentz model [25]: $\varepsilon_{\mathrm{Si}}(\omega)=$ $1+\sum_{i=1}^{4}\left(A_{i} /\left(\omega-\omega_{i}\right)-A_{i}^{*} /\left(\omega+\omega_{i}^{*}\right)\right)$ with $\omega_{1}=(7.99-1.83 i) \times$ $10^{15} \mathrm{rad} / \mathrm{s}, \quad \omega_{2}=(0.788+0.134 i) \omega_{1}, \quad \omega_{3}=(0.671+0.07 i) \omega_{1}$, $\omega_{4}=(0.613+0.12 i) \omega_{1}, \quad A_{1}=(-1.53-0.624 i) \omega_{1}, \quad A_{2}=$ $(-1.17-0.778 i) \omega_{1}, \quad A_{3}=(-1.15+0.565 i) \omega_{1}, \quad$ and $A_{4}=$ $(-0.440+0.216 i) \omega_{1}$. The silver permittivity is approximated by a single-pole Drude model $\varepsilon_{\mathrm{Ag}}(\omega)=\varepsilon_{\infty}-\varepsilon_{\infty} \omega_{p}^{2} /\left(\omega^{2}+i \omega \gamma\right)$ with $\varepsilon_{\infty}=1, \omega_{p}=1.37 \times 10^{16} \mathrm{rad} / \mathrm{s}$, and $\gamma=0.0023 \omega_{p}$.

a Lanczos-type algorithm, which may compute several eigenpairs at one time of calculation. Because the Arnoldi method preferentially computes the modes with the largest eigenvalue magnitudes [27], in ARPACK, a shift and invert spectral transformation is implemented, so that only the eigenmodes in the concerned interval of the spectrum are returned. In our work, we chose the solver MUMPS [28], a parallel sparse direct solver based on LU decomposition, to perform the spectrum transformation.

In the present work, the spectral transformation is performed such that the ARPACK solver first finds eigenvectors with eigenfrequencies close to $f_{p}=5 \times 10^{14} \mathrm{~Hz}$, a central frequency of the spectral interval of interest. We asked the solver to return 30 modes in total. We use a perfect-magnetic-conductor boundary condition in the $(\rho, z)$ plane containing the emitter, 
and, thanks to this symmetry, i.e., the calculation per QNM, generally lasts $0.8 \mathrm{~min}$ on our desktop computer (Intel Xeon Processor E5-2643) with $\approx 7.6 \times 10^{5}$ degrees of freedom for the mesh. The per-mode computational speed is determined by many factors, e.g., the frequency $f_{p}$, the density of eigenmodes, and the number of the eigenmodes that are computed. We empirically observe that when only two to three modes are computed, the per mode computation time is about $5 \mathrm{~min}$. However, once more modes are returned, the computational time per mode is readily reduced to less than $1 \mathrm{~min}$, which is similar to the CPU time required for performing one real frequency simulation.

The frequency positions in the complex-frequency plane of the 30 modes are shown in Fig. 1(b). Note that the exp $(-i \omega t)$ convention is used throughout the article for all the figures and formulas. We compute more modes than needed for the reconstruction to guarantee that all the relevant QNMs are found. However, computing additional modes is not a pure waste of time. They contribute to improve the accuracy of the reconstruction, as will be discussed in the following. Furthermore, they can be useful for computing the nanoresonator response for other driving currents, for which the additional modes may become quite relevant. Finally, in an iterative inverse-design approach based on an ultrafast computation of the QNMs using high-order QNM perturbation theory, these additional modes may reinforce the accuracy of the approach, even when large (up to $50 \%$ in volume) deformations are considered to rapidly span the parameter space [29].

In the second step, we calculate the excitation coefficient of every individual mode with a closed-form expression [24]:

$$
\alpha_{m}(\omega)=\frac{\omega}{\tilde{\omega}_{m}-\omega} \tilde{\mathbf{E}}_{m}\left(\mathbf{r}_{0}\right) \cdot \mathbf{p}
$$

$\tilde{\mathbf{E}}_{m}$ being the normalized electric field of the $m$ th QNM, $\tilde{\omega}_{m}$ its QNM complex frequency, $\mathbf{p}=p \mathbf{e}_{z}$ with $p=1 \mathrm{C} \cdot \mathrm{m}$ the dipole moment placed at position $\mathbf{r}_{0}$. Note that analytical expressions, similar to Eq. (1), also exist for far-field illumination [30].

The total field $\mathbf{E}(\mathbf{r})$ radiated by the source at the real frequency $\omega$ is then reconstructed as

$$
\mathbf{E}^{(0)}(\mathbf{r})=\sum_{m=1}^{M} \alpha_{m}(\omega) \tilde{\mathbf{E}}_{m}(\mathbf{r}) .
$$

Note the superscript (0) introduced in the left-hand term. Its significance will become clear when we introduce interpolation points. The expansion of the field converges toward the exact result as the truncation rank $M$ tends toward infinity due to completeness of the basis formed by the QNMs and the numerical modes [8-10,22]. Among the 30 modes, five modes have a spectrally integrated excitation coefficient, $\bar{A}_{m}=\int_{\lambda_{s}}^{\lambda_{e}}\left|\alpha_{m}(2 \pi c / \lambda)\right|^{2} \mathrm{~d} \lambda$, which is much larger than the other modes. They will be considered as the true physical QNMs that dominantly impact the reconstruction. We have also checked that their complex frequencies are nearly independent of the PMLs used in the computation [9]. Their normalized fields, e.g., the electric-field $z$ component of $\operatorname{Re}\left(\tilde{E}_{m, z}\right)$, are highly confined beneath the dielectric cylinder, indicating that they are Fabry-Perot resonances formed by the bouncing of gap plasmons [see Fig. 1(c)].

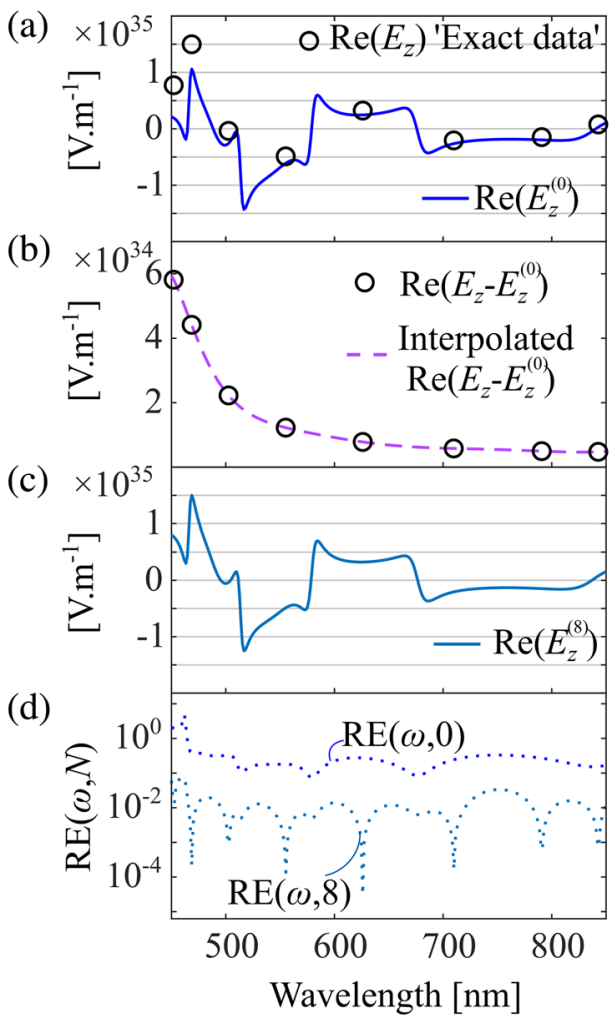

Fig. 2. Step-by-step presentation of the hybrid method for reconstructing the spectrum of $\operatorname{Re}\left(E_{z}(x, 0,0)\right)$ at point $P(x=-53 \mathrm{~nm})$. (a) Precomputed data before interpolation. Black circles represent "exact" data $\operatorname{Re}\left(E_{z}\right)$ computed by performing light-scattering simulations at eight Chebyshev points; blue curve corresponds to the QNM reconstruction $\operatorname{Re}\left(E_{z}^{(0)}\right)$ with $M=30$ QNMs. (b) Difference between the exact data $\operatorname{Re}\left(E_{z}\right)$ and the reconstructed result $\operatorname{Re}\left(E_{z}^{(0)}\right)$. The violet curve is interpolated from the circles. (c) The interpolated result $\operatorname{Re}\left(E_{z}^{(8)}\right)$ obtained as the sum of the blue curve in (a) and violet curve in (b). (d) Relative errors of the reconstructed result, without, $\operatorname{RE}(\omega, 0)$, and with interpolation, $\operatorname{RE}(\omega, 8)$.

In Fig. 2(a), the solid curve shows the reconstructed spectrum of the $z$ component of the radiated electric field computed at the mirror-image point of the emitter [the point is denoted by "P" in Fig. 1(a)]. $M=30$ modes are used for the reconstruction. However, only the five QNMs with the largest $\bar{A}_{m}$ significantly contribute to the reconstructed spectrum with sharp variations, the contribution of the other 25 modes being essentially flat spectrally.

\section{B. Interpolation with Real-Frequency Simulation Results}

Indeed, a large number of irrelevant modes with low-quality factors or frequencies outside the spectral window are expected to smoothly contribute to the reconstruction in a non-negligible manner. The accuracy of the reconstruction can be increased by increasing the truncation rank $M[8-10]$ or by using a contour integral in the complex-frequency plane [21]. Instead, we simply complement the truncated QNM reconstruction with a few real-frequency computational data obtained with lightscattering simulations. These real-frequency results are obtained with COMSOL Multiphysics, but they can also be obtained 
with any frequency-domain numerical method. The data are shown with circles in Fig. 2(a) and are considered as "exact" data because their accuracy can be made arbitrarily high by refining the mesh.

The real-frequency computational data slightly differ from the QNM prediction spectra. The difference,

$$
u(\omega)=E_{z}(\omega)-E_{z}^{(0)}(\omega),
$$

is shown with circles in Fig. 2(b). In the spectral range $\left[\omega_{s}, \omega_{e}\right]$ of interest, the difference $u(\omega)$ is found to be a slowly varying function of the frequency. Thus, it can be approximated with a polynomial expansion, $u(\omega) \approx u^{(N)}(\omega)=$ $\sum_{k=1}^{N} u\left(\omega_{k}\right) \varphi_{k}(\omega)$ [31]. We use the Lagrange polynomials $\varphi_{k}(\omega)=\prod_{j=1, j \neq k}^{N}\left(\omega-\omega_{j}\right) /\left(\omega_{k}-\omega_{j}\right)$, but the choice is rather unimportant as discussed hereafter. For the interpolation points at frequency $\omega_{k}, k=1,2, \ldots, N$, one may rely on regularly spaced interpolation points and use a spline or cubic interpolation, as proposed in the MATLAB software, for instance. Here, we adopt Chebyshev points, but similar convergence has been obtained with other interpolation methods (see Section 3 for details). As $N$ increases, the solution converges toward the exact solution.

By fitting $u(\omega)$ with only $N=8$ Chebyshev points, a faithful prediction $E_{z}^{(N)}=u^{(N)}(\omega)+E_{z}^{(0)}$ of $E_{z}=u(\omega)+E_{z}^{(0)}$ can be readily obtained [see the solid curve in Fig. 2(c)]. To quantify the precision of the interpolation, we define the relative error

$$
\operatorname{RE}(\omega, N)=2\left|\frac{F(\omega)-F^{(N)}(\omega)}{F(\omega)+F^{(N)}(\omega)}\right|,
$$

where $F$ stands for an arbitrary field component or physical quantity obtained with direct solutions of the scattering problem, and $F^{(N)}$ is the result reconstructed with $M$ modes plus interpolation data with $N$ Chebyshev points. Such a definition equally handles large $F$ data as well as small. With the direct solver MUMPS implemented in COMSOL Multiphysics, we have computed $F=E_{z}$ for 300 frequencies regularly spaced in the interval $\left[\omega_{s}, \omega_{e}\right]$. As can be seen in Fig. 2(d), without interpolation, the relative error $\operatorname{RE}(\omega, N=0)$ is $\approx 10^{-1}$. On the other hand, $\operatorname{RE}(\omega, N=8)$ remains smaller than a few percent almost over the entire spectral range. We will see that the relative error rapidly decreases with $N$. The enhanced error at short wavelengths is mainly due to the existence of many high-frequency QNMs that contribute to the response in the blue. As we shall see in Section 3, their impact, which is directly observed in Fig. 2(b), can be drastically reduced by intentionally adding more interpolation points at short wavelengths $(2 \pi c / \omega<500 \mathrm{~nm})$. Alternatively, it may also be leveraged by considering more QNMs in this spectral range.

\section{IMPLEMENTATION AND CONVERGENCE TESTS}

Interpolation methods are well-documented in the literature [32]. Their predictive force strongly depends on the "smoothness" of the function. For periodic structures, e.g., gratings, the field is continuous with the frequency $\omega$ but not derivable at cutoff frequencies, where a propagating diffracted order becomes evanescent. Otherwise, for resonators with finite sizes, the electromagnetic fields have continuous derivatives with the frequency $\omega$ of all orders [33]. Therefore, we do not anticipate important difficulties with the interpolation.

We first study the convergence of the result obtained with Chebyshev points $\omega_{k}=\omega_{s}+\left(1+x_{k}\right)\left(\omega_{e}-\omega_{s}\right) / 2$, $x_{k}=\cos [(2 k-1) \pi /(2 N)]$ being the points in the interval $[-1,1]$. The approach avoids large oscillations of the sum of the Lagrange polynomials that are observed with even point distribution at the edges of the interpolation interval (Runge's phenomenon). Furthermore, its performance is welldocumented [34]. Note that the Runge's phenomenon may also be avoided by using Clenshaw-Curtis points or Leja points.

Figure 3(a) shows the convergence of the relative error $\operatorname{RE}(\omega, N)$ for the field $F=E_{z}(x, y)$ in the median plane of the gap $z=0$ for a wavelength of $565 \mathrm{~nm}$, which does not correspond to any frequency of the discrete set of Chebyshev points. The first two panels show the "exact" results for $\operatorname{Re}\left(E_{z}\right)$ and $\operatorname{Im}\left(E_{z}\right)$. Three values of $N$ are considered, $N=0$ (QNM reconstruction without interpolation), $N=8$, and $N=20$. The relative error is reduced by more than a factor of 10 for $N=8$, and, from the rightmost panel, we see that the error is further reduced by another factor of 10 for $N=20 . \operatorname{RE}(\omega, 20)$ is small almost everywhere. The maximum values $\approx 10^{-2}$ are obtained at locations where $\left|E_{z}\right| \approx 0$. Note that it is difficult to define a relative error for small $F$ values. With the conventional definition $\left|\left(F-F^{(N)}\right) / 2 F\right|$, the relative error is infinite for $F=0$, even for arbitrarily small (thus highly accurate) values of $F^{(N)}$. With the definition of Eq. (4), this value is always 2 .

To quantitatively measure the convergence performance, we consider a spectrally and spatially averaged relative error denoted as $\langle\mathrm{RE}\rangle$, where $\langle\cdot\rangle$ denotes spectral and spatial averaging, respectively, performed over 300 frequencies uniformly distributed in the spectral interval $\left[\omega_{s}, \omega_{e}\right]=[2 \pi c / 850 \mathrm{~nm}, 2 \pi c / 450 \mathrm{~nm}]$ and over $11 \times 11$ points of a centered square of size $D^{2}(D$ being the diameter of the Si cylinder) placed in the median plane of the gap. The curve "Che" in Fig. 3(b) shows $\langle\mathrm{RE}\rangle$ for increasing values of $N$ and for Chebyshev points. The averaged error $\langle\mathrm{RE}\rangle$ follows the same trend as that presented in Fig. 3(a), reaching $10^{-2}$ for $N=8$ and $10^{-3}$ for $N=20$. Further increasing $N$ leads to a relatively slower decrease of $\langle\mathrm{RE}\rangle$, as can be seen from the inset in Fig. 3(b). We do not precisely know the origin of the two-slope convergence observed in the inset Fig. 3(b). We have tested the interpolation approach on slowly varying analytical $\left(C^{\infty}\right)$ functions, e.g., sine, and we observed the same behavior, with a faster convergence rate nevertheless.

Besides interpolation with Lagrange polynomials, we have also tried the cubic and spline methods with MATLAB to interpolate the Chebyshev points. The corresponding values of $\langle\mathrm{RE}\rangle$ are shown with the curves labeled "Spline Che" and "Cubic Che." The convergence rates of "Spline Che" and "Che" are similar. A slightly better convergence is observed for the cubic interpolation labeled "Cubic Che" for small values of $N$.

Chebyshev points change with increasing $N$, implying for instance that the set of $\omega_{k}$ for $N+1$ points is completely different from the set of $\omega_{k}$ for $N$ points. This is a drawback. If one decides to add a new interpolation point to increase the accuracy, real-frequency simulations should be performed again for all new sets of points. Thus, in practice, it is convenient to consider recursive interpolation schemes that allow us to 
(a)

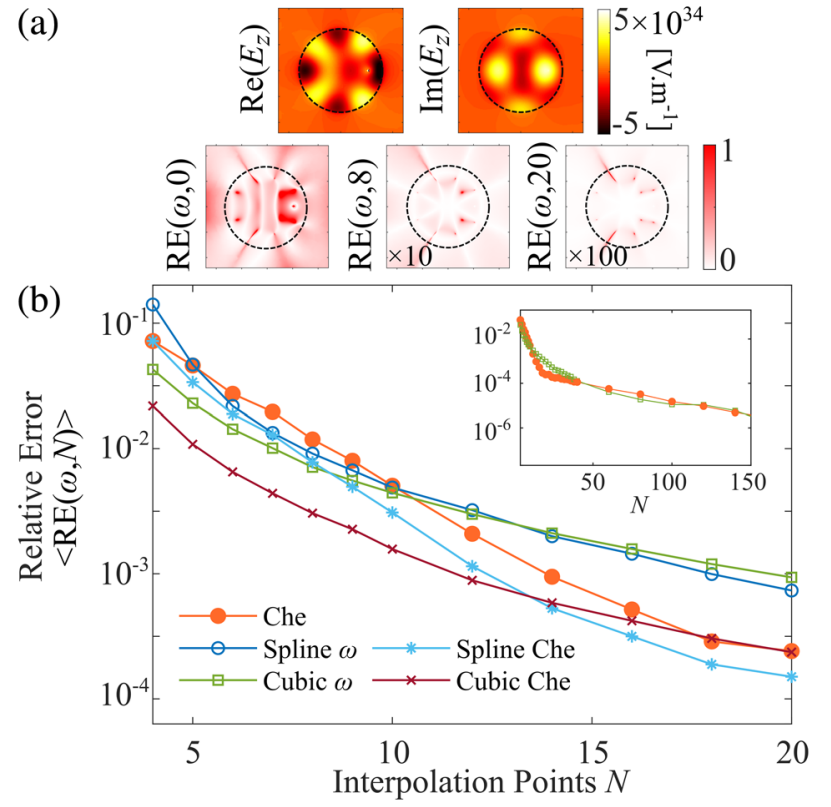

Fig. 3. (a) Upper panels: $E_{z}$ in the plane $z=0$ at $\lambda=562 \mathrm{~nm}$. Lower panels: Relative error RE of the reconstructed field for $N=0$, 8, and 20. $M=30$ QNMs are used in the expansion of Eq. (2). $N=0$ corresponds to the QNM reconstruction without interpolation. (b) Spatially and spectrally averaged relative error $\langle\mathrm{RE}\rangle$ of $E_{z}^{(N)}$ as a function of $N$ for five interpolation methods: "Che," "Spline Che," and "Cubic Che" are obtained for Chebyshev points using the Lagrange polynomials, spline, and cubic methods, respectively; "Spline $\omega$ " and "Cubic $\omega$ " are obtained for uniformly distributed frequency points using the spline and cubic methods, respectively. Inset shows the convergence of the "Che" and "Cubic $\omega$ " methods up to $N=150$.

increase $N$ by reusing available interpolation points. Thus, we consider cubic and spline interpolation schemes with interpolation points uniformly distributed in the spectral interval $\left[\omega_{s}, \omega_{e}\right]$. The convergence of these methods is shown with the curves labeled with "Spline $\omega$ " and "Cubic $\omega$ " in Fig. 3(b). Interestingly, from the inset, we find that the convergence rates of the "Spline $\omega$ " and "Che" methods are similar for large $N$. On the other hand, for small $N$, the relative errors of these two methods are larger compared with those relying on Chebyshev points. This is simply due to the fact that the Chebyshev points are more densely distributed for small wavelengths for which the difference $u(\omega)=E_{z}(\omega)-E_{z}^{(0)}(\omega)$ varies more rapidly.

\section{APPLICATION}

In cavity-quantum-electrodynamic experiments, the exact position or polarization of the emitter is rarely known with accuracy, and the emission spectrum might be broad. Therefore, a quantitative interpretation of the experimental results often requires many electromagnetic simulations to explore a large parameter space. Inspired by this example, this section aims at quantifying the strengths and weaknesses of the hybrid method. Specifically, we consider the variation of the normalized decay rate $\gamma / \gamma_{0}=\frac{2}{\hbar \gamma_{0}} \operatorname{Im}\left[\mathbf{p}^{*} \cdot \mathbf{E}\left(\mathbf{r}_{0}\right)\right]$ with the frequency $\omega$ and position of the emitter $\mathbf{r}_{0}$, where $\mathbf{E}\left(\mathbf{r}_{0}\right)$ is the field radiated by the dipole and $\gamma_{0}=\omega^{3}|\mathbf{p}|^{2} /\left(3 \pi \varepsilon_{0} \hbar c^{3}\right)$ is the spontaneous decay rate of the same dipole in free space [24]. We restrict the study to vertically polarized dipoles positioned in the median plane of the gap; other polarizations and positions may be similarly considered, however. We denote by $N_{\lambda}$ and $N_{r_{0}}$ the number of wavelengths and emitter positions considered to span the parameter space.

Figure 4(a) shows the QNM reconstruction of $\gamma^{(0)} / \gamma_{0}$ obtained with $M=30$ QNMs, computed for $N_{r_{0}}=20 x$ positions of the emitter and, for each position, $N_{\lambda}=300$ wavelengths. Consequently, 6000 different instances of the dipole position and frequency are considered. The QNM reconstruction is fast and does not depend on $N_{\lambda}$ and $N_{r_{0}}$, owing to the availability of closed-form expressions [see Eqs. (1) and (2)]. Typically, the CPU time to compute one QNM being $\approx 1 \mathrm{~min}$, the whole decay-rate map is obtained in $\tau=30 \mathrm{~min}$. We recall that a single symmetry plane is used in the computation. Reconstructions with QNM expansions may provide highly accurate predictions of the decay rate. For instance, they may quantitatively predict quenching, an effect of great significance that limits the brightness of the nanoresonators. However, the quenching prediction with QNM expansion is rarely met in the literature [9], as it remains challenging even for advanced QNM solvers to compute several hundreds of high-order QNMs. The present approach solves this problem: Because quenching is a slowly varying function of the frequency, it is accounted for with the interpolation step.

From Fig. 4(a), we see that the decay-rate spectra of off-axis emitters are characterized by several sharp peaks. This indicates that the responses are dominated by a few QNMs with high-quality factors. On the other hand, the spectra of nearly on-axis emitters show broad resonance peaks. The latter is due to QNMs with small azimuthal numbers. These QNMs have relatively low-quality factors and high radiative loss.

Figure 4(b) shows the normalized decay rate $\gamma^{(7)} / \gamma_{0}$ map using seven Chebyshev points for each of the $N_{r_{0}}=20$ emitter positions. This step is the most demanding in the computational resources. Because the estimated CPU time for each real-frequency simulation is $\approx 1 \mathrm{~min}$ (as a rule of thumb, we have noted that the CPU times to compute one QNM or to run one real-frequency simulation with the same mesh are nearly identical); in addition, the $7 \times 20$ real-frequency simulations are performed in $\approx 2 \mathrm{~h}$. By contrast, computing the 6000 different instances for the same map with fully vectorial frequency-domain computation would require almost four days.

We also test the accuracy of the present method by comparing the predictions with those obtained with "exact" reference data obtained with real-frequency simulations performed with COMSOL Multiphysics. Two emitter positions are considered. For $x_{0}=53 \mathrm{~nm}$, the total decay-rate spectrum is strongly impacted by four high-Q QNMs. Reversely, a smooth spectral response is obtained for $x_{0}=27 \mathrm{~nm}$. Figure 4(c) shows the relative errors $\operatorname{RE}(\omega, 7)$ for both positions. The error is kept below $3.2 \times 10^{-2}$ over the full spectral range. $\operatorname{RE}(\omega, 7)$ for the $x_{0}=53 \mathrm{~nm}$ is relatively larger, as an off-axis emitter is capable of exacting more modes compared with an on-axis emitter. We have observed that, for a larger set of QNMs, the relative errors become equal and significantly smaller $\left(\operatorname{RE}(\omega, 7)<8 \times 10^{-3}\right.$ for $M=120)$. 

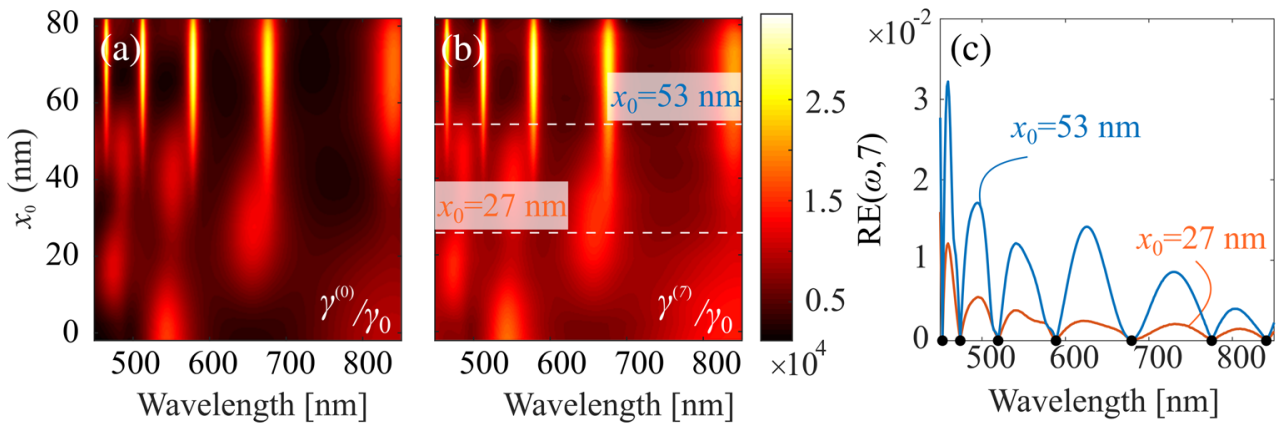

Fig. 4. Reconstruction maps of the total decay rate spectrum $\gamma(\omega) / \gamma_{0}$ of single emitters placed at various positions on the $x$ axis. Only positive $x$ values are considered for symmetry reasons. (a) Reconstruction obtained with $M=30$ QNMs without interpolation. The CPU times of $\tau=30$ min are independent of $N_{\lambda}$ and $N_{r_{0}}$. (b) Interpolated results $\gamma^{(7)} / \gamma_{0}$ with seven Chebyshev points. $N_{r_{0}}=20 x$ coordinates are considered, requiring a total CPU time of $20 \times 7=140 \mathrm{~min}$. (c) Relative error $\operatorname{RE}(\omega, 7)=2\left|\left(\gamma^{(7)}-\gamma\right) /\left(\gamma^{(7)}+\gamma\right)\right|$ for two emitter positions: $x_{0}=53 \mathrm{~nm}$ and $27 \mathrm{~nm}$. Chebyshev points are shown with the black dots.

Table 1. CPU Times Estimated for Computing $\gamma / \gamma_{0}$ with an Intel Xeon Processor E5-2643 with $\approx 3.2 \times 10^{5}$ Degrees of Freedom for the Real-Frequency Simulation and $7.6 \times 10^{5}$ for the QNM Computation ${ }^{b}$

\begin{tabular}{|c|c|c|c|c|c|c|c|}
\hline$N_{\lambda}$ & $N_{r_{0}}$ & $\begin{array}{c}\text { QNM } \\
\text { Reconstruction } \\
M=\mathbf{3 0}\end{array}$ & $\begin{array}{c}\text { Interpolation } \\
\qquad N=7\end{array}$ & $\begin{array}{c}\text { Total } M=30, \\
N=7 \text { (Relative } \\
\left.\text { Error }^{a}<3.2 \%\right)\end{array}$ & $\begin{array}{l}\text { Total } M=120 \\
N=7 \text { (Relative } \\
\left.\text { Error }^{a}<\mathbf{0 . 8} \%\right)\end{array}$ & $\begin{array}{c}\text { Total } M=30, \\
N=9 \text { (Relative } \\
\left.\text { Error }^{a}<0.7 \%\right)\end{array}$ & $\begin{array}{c}\text { "Exact" } \\
\text { Real-Frequency } \\
\text { Simulations } \\
\end{array}$ \\
\hline 1001 & 20 & $30 \mathrm{~min}$ & $140 \mathrm{~min}$ & $2.8 \mathrm{~h}$ & $4.7 \mathrm{~h}$ & $3.5 \mathrm{~h}$ & 14 days \\
\hline 101 & 200 & $30 \mathrm{~min}$ & $23 \mathrm{~h}$ & $24 \mathrm{~h}$ & $25 \mathrm{~h}$ & $30 \mathrm{~h}$ & 14 days \\
\hline
\end{tabular}

${ }^{a}$ Estimated for $x_{0}=53 \mathrm{~nm}$.

${ }^{b}$ The numbers of mesh elements used for the two kinds of simulations are the same. The difference between the degrees of freedom is due to the use of the auxiliary fields in the QNM computation.

Based on these observations, Table 1 summarizes our estimations of the CPU times required to fully study the emission spectrum of a localized dipole for several values of $N_{\lambda}$ and $N_{r_{0}}$. Comparison with "exact" data obtained with real-frequency simulations shows that the present method may provide significant CPU accelerations. Since the latter strongly depends on $N_{\lambda}$ and $N_{r_{0}}$, we let the readers draw their own opinions.

\section{DISCUSSION}

For the sake of fairness, we stress that the CPU times for the "Exact" real frequency simulations in Table 1 are estimated for the cases where the frequency sweepings are conducted with brute force. By utilizing more advanced techniques, such as the block flexible restarted GMRES [35], a method designed for solving large linear systems of equations with several right-hand sides, e.g., Maxwell's equations with multiple excitations, much better computational efficiencies can be achieved. Therefore, the CPU-time saving of the present method may be overestimated, especially for scientists using or developing advanced numerical tools. However, the present method that addresses the slow convergence issue of the modal expansion method can be particularly useful for a broad audience relying on commercial software, which does not implement advanced matrix solvers. The simplicity of the method may even allow the readers to easily implement it by downloading the models in MAN [23]. Furthermore, note that the computational speed of our method can also be benefit from the utilization of advanced matrix-solving techniques to significantly reduce the CPU time for the computation of the interpolation points. How the present method may benefit from the advanced matrix-solving techniques is a likely direction for future work.

Because the acceleration is primarily due to the predictive force of the QNM expansion that provides an analytical treatment for the frequency dependence, it is natural to wonder how the present method compares with the finite-difference time-domain (FDTD) method. Such comparisons between completely different methods are always difficult to make fairly [36]. They significantly depend on the material dispersion that must be included for realistic modeling and, additionally, on the geometry itself and on the capability to accurately mesh deep subwavelength domains with rounded corners. The present results are obtained with an element polynomial degree of 2 , but higher order is available and with a careful meshing of the $5 \mathrm{~nm}$ thin silver film and the $2 \mathrm{~nm}$ thin air gap. We believe that achieving a similar accuracy with the FDTD method requires advanced implementation skills. For comparison, one should also consider that the FDTD method requires repeating the entire computation whenever one considers different instances of the driving field, such as the pulse shape or duration, polarization, or incidence angle. This is not the case with the present approach, at least for the first computational step leading to the QNM reconstruction. Furthermore, a fair comparison would also require considering, in addition to computational speed and accuracy, the gain of the physical insight brought by the QNM expansion used in the present approach.

The hybrid scheme that combines complex and real frequency simulations is mostly dictated by simple intuition: 
The incorporation of a few "exact" data regularly sampled in a given spectral interval should enhance the accuracy of the reconstruction. The approach leads to abrupt dips in the relative error curve, exemplified by the curve $\operatorname{RE}(\omega, 8)$ in Fig. 2(d). A more effective approach may consist of replacing the realfrequency points by complex-frequency points (computation at complex frequencies can be easily performed with COMSOL by renormalizing the permittivity and permeability, see [30]) chosen close to the real axis in the upper-half complex- $\omega$ plane, where the field is analytical owing to causality [37]. Exact data at complex frequencies are expected to represent a spectrally averaged response over a spectral range given by the imaginary part of the complex frequency [38]. They may regularize the interpolation over the whole spectral range, replacing the dips by a smoother curve. We thus anticipate that the intuitive hybrid scheme proposed in this work can be further refined. There are also opportunities for accelerating the method. In the present implementation, the QNM and real-frequency computations are performed completely independently. However, since the rate of convergence of iterative solvers is impacted by the condition number of the shifted system, knowing the QNMs in the interval of interest and including them in the Krylov subspace may enhance the effective condition number and reduce the computation loads for the interpolation points.

\section{CONCLUSION}

We have presented a hybrid approach for accurately and effectively analyzing microcavities and nanoresonators. The approach combines the respective forces of real- and complexfrequency simulations. It alleviates difficulties encountered with purely frequency approaches, for which the lack of initial knowledge on the resonance frequencies imposes a fine sampling in the frequency domain for carefully displaying the linewidths and amplitudes of the resonance peaks, especially for high- $Q$ dielectric systems. It also gets around difficulties encountered with QNM expansion techniques, which may require a great number of QNMs and numerical modes to achieve high accuracy.

The effectiveness of the method is provided by the analyticity of the QNM expansion. Its accuracy results from the interpolation performed with a few additional real-frequency simulations. More importantly, the method preserves the force of modal-expansion approaches to provide a physically transparent interpretation of all the salient features of the resonator response.

The hybrid method is implemented in the freeware MAN, which relies on COMSOL Multiphysics. It benefits from the versatility of the QNM Solver QNMEig [9], which can be applied to a variety of geometries and materials, even with complicated dispersion relation, as is the case for the nanoresonator analyzed in the present work. The user may further choose the interpolation method (spine, cubic, Chebyshev...) and define the number of real-frequency interpolation points and number of QNMs used for the initial reconstruction without interpolation. We expect that the hybrid method may contribute to the deployment of efficient inverse design tools, benefiting from the possibilities offered by QNM expansions to accelerate computations and interpret the results.
Funding. Deutsche Forschungsgemeinschaft (EXC-2046/1).

Acknowledgment. A. Gras acknowledges the support of the DGA and INRIA. F. Binkowski acknowledges the support of the German Research Foundation via EXC-2046/1, project ID: 390685689. The authors further acknowledge interesting discussions with the reviewers.

Disclosures. The authors declare no conflicts of interest.

Data Availability. Data underlying the results presented in this paper are not publicly available at this time but may be obtained from the authors upon reasonable request.

\section{REFERENCES}

1. P. Lalanne, W. Yan, V. Kevin, C. Sauvan, and J.-P. Hugonin, "Light interaction with photonic and plasmonic resonances," Laser Photon. Rev. 12, 1700113 (2018).

2. J. S. Jensen and O. Sigmund, "Topology optimization for nano-photonics," Laser Photon. Rev. 5, 308 (2011).

3. K. Yao, R. Unni, and Y. Zheng, "Intelligent nanophotonics: merging photonics and artificial intelligence at the nanoscale," Nanophotonics 8, 339-366 (2019).

4. S. Molesky, Z. Lin, A. Y. Piggott, W. Jin, J. Vucković, and A. W. Rodriguez, "Inverse design in nanophotonics," Nat. Photonics 12, 659-670 (2018).

5. I. Malkiel, M. Mrejen, A. Nagler, U. Arieli, L. Wolf, and H. Suchowski, "Plasmonic nanostructure design and characterization via deep learning," Light Sci. Appl. 7, 60 (2018).

6. P. Wiecha, A. Arbouet, C. Girard, and O. Muskens, "Deep learning in nano-photonics: inverse design and beyond," Photon. Res. 9, B182B200 (2021).

7. W. Ma, Z. Liu, Z. A. Kudyshev, A. Boltasseva, W. Cai, and Y. Liu, "Deep learning for the design of photonic structures," Nat. Photonics 15, 77-90 (2021).

8. B. Vial, F. Zolla, A. Nicolet, and M. Commandré, "Quasimodal expansion of electromagnetic fields in open two-dimensional structures," Phys. Rev. A 89, 023829 (2014).

9. W. Yan, R. Faggiani, and P. Lalanne, "Rigorous modal analysis of plasmonic nanoresonators," Phys. Rev. B 97, 205422 (2018).

10. A. Gras, P. Lalanne, and M. Duruflé, "Non-uniqueness of the quasinormal mode expansion of electromagnetic Lorentz dispersive materials," J. Opt. Soc. Am. A 37, 1219-1228 (2020).

11. A. Gras, W. Yan, and P. Lalanne, "Quasinormal-mode analysis of grating spectra at fixed incidence angles," Opt. Lett. 44, 3494-3497 (2019).

12. E. A. Muljarov and W. Langbein, "Exact mode volume and Purcell factor of open optical systems," Phys. Rev. B 94, 235438 (2016).

13. C. Sauvan, "Quasinormal modes expansions for nanoresonators made of absorbing dielectric materials: study of the role of static modes," Opt. Express 29, 8268-8282 (2021).

14. F. Zolla, A. Nicolet, and G. Demésy, "Photonics in highly dispersive media: the exact modal expansion," Opt. Lett. 43, 5813-5816 (2018).

15. C. Gigli, T. Wu, G. Marino, A. Borne, G. Leo, and P. Lalanne, "Quasinormal-mode non-Hermitian modeling and design in nonlinear nano-optics," ACS Photon. 7, 1197-1205 (2020).

16. S. Jahani, A. Roy, and A. Marandi, "Wavelength-scale optical parametric oscillators," Optica 8, 262-268 (2021).

17. S. D. Rezaei, Z. Dong, J. Y. E. Chan, J. Trisno, R. J. H. Ng, Q. Ruan, C. W. Quu, N. A. Mortensen, and J. K. W. Yang, "Nanophotonic structural colors," ACS Photon. 8, 18-33 (2021).

18. Q. Li, T. Wu, J. van de Groep, P. Lalanne, and M. L. Brongersma, "Structural color from a coupled nanowire pair beyond the bonding and antibonding model," Optica 8, 464-470 (2021).

19. D. Navarro-Urrios, N. E. Capuj, M. F. Colombano, P. D. García, M. Sledzinska, F. Alzina, A. Griol, A. Martínez, and C. M. SotomayorTorres, "Nonlinear dynamics and chaos in an optomechanical beam," Nat. Commun. 8, 14965 (2017).

20. J. Ng, Z. Lin, and C. T. Chan, "Theory of optical trapping by an optical vortex beam," Phys. Rev. Lett. 104, 103601 (2010). 
21. L. Zschiedrich, F. Binkowski, N. Nikolay, O. Benson, G. Kewes, and S. Burger, "Riesz-projection-based theory of light-matter interaction in dispersive nanoresonators," Phys. Rev. A 98, 043806 (2018).

22. F. Binkowski, F. Betz, R. Colom, M. Hammerschmidt, L. Zschiedrich, and S. Burger, "Quasinormal mode expansion of optical far-field quantities," Phys. Rev. B 102, 035432 (2020).

23. "QNM solvers and toolboxes are freely available at the webpage of the group of the last author here; we modified the weak form of COMSOL, so that the QNM for a resonator with the multipole permittivity can be solved," https://www.lp2n.institutoptique.fr/lightcomplex-nanostructures.

24. C. Sauvan, J. P. Hugonin, I. S. Maksymov, and P. Lalanne, "Theory of the spontaneous optical emission of nanosize photonic and plasmon resonators," Phys. Rev. Lett. 110, 237401 (2013).

25. M. Garcia-Vergara, G. Demésy, and F. Zolla, "Extracting an accurate model for permittivity from experimental data: hunting complex poles from the real line," Opt. Lett. 42, 1145-1148 (2017).

26. Y. Yang, O. D. Miller, T. Christensen, J. D. Joannopoulos, and M. Soljačić, "Low-loss plasmonic dielectric nanoresonators," Nano Lett. 17, 3238-3245 (2017).

27. R. B. Lehoucq, D. C. Sorensen, and C. Yang, ARPACK Users' Guide: Solution of Large Scale Eigenvalue Problems with Implicitly Restarted Arnoldi Methods (Rice University, 1997).

28. P. Amestoy, I. Duff, J. Koster, and J.-Y. L'Excellent, "A fully asynchronous multifrontal solver using distributed dynamic scheduling," SIAM J. Matrix Anal. Appl. 23, 15-41 (2001).
29. W. Yan, P. Lalanne, and M. Quu, "Shape deformation of nanoresonator: a quasinormal-mode perturbation theory," Phys. Rev. Lett. 125, 013901 (2020).

30. Q. Bai, M. Perrin, C. Sauvan, J.-P. Hugonin, and P. Lalanne, "Efficient and intuitive method for the analysis of light scattering by a resonant nanostructure," Opt. Express 21, 27371-27382 (2013).

31. J. Stoer and R. Bulirsch, Introduction to Numerical Analysis (SpringerVerlag, 2002), p. 73.

32. J. P. Boyd, Chebyshev and Fourier Spectral Methods (Dover Publications, 2001), p. 84.

33. A. Habib, personal communication (2021).

34. P.-J. Laurent, Approximation et Optimisation (Hermann, 1972).

35. H. Calandra, S. Gratton, J. Langou, X. Pinel, and X. Vasseur, "Flexible variants of block restarted GMRES methods with application to geophysics," SIAM J. Sci. Comput. 34, A714-A736 (2012).

36. T. Grosges, A. Vial, and D. Barchiesi, "Models of near-field spectroscopic studies: comparison between finite-element and finite-difference methods," Opt. Express 13, 8483-8497 (2005).

37. L. D. Landau, L. P. Pitaevskii, and E. M. Lifshitz, Electrodynamics of Continuous Media, 2nd ed. (Butterworth-Heinemann, 1984).

38. X. Liang and S. G. Johnson, "Formulation for scalable optimization of microcavities via the frequency-averaged local density of states," Opt. Express 21, 30812-30841 (2013). 